\title{
Feasibility of low-dose digital pulsed video-fluoroscopic swallow exams (VFSE): effects on radiation dose and image quality
}

Acta Radiologica

2017, Vol. 58(9) 1037-1044

(C) The Foundation Acta Radiologica 2017

Reprints and permissions:

sagepub.co.uk/journalsPermissions.nav DOI: $10.1|77 / 0284| 85 \mid 16685924$ journals.sagepub.com/home/acr @SAGE

\author{
Jakob Weiss ${ }^{\text {I,2 }}$, Mike Notohamiprodjo ${ }^{1,2}$, Klement Neumaier ${ }^{3}$, \\ Minglun $\mathrm{Li}^{3}$, Wilhelm Flatz ${ }^{2}$, Konstantin Nikolaou ${ }^{1,2}$ and \\ Andreas Pomschar ${ }^{2}$
}

\begin{abstract}
Background: Fluoroscopy is a frequently used examination in clinical routine without appropriate research evaluation latest hardware and software equipment.

Purpose: To evaluate the feasibility of low-dose pulsed video-fluoroscopic swallowing exams (pVFSE) to reduce dose exposure in patients with swallowing disorders compared to high-resolution radiograph examinations (hrVFSE) serving as standard of reference.

Material and Methods: A phantom study (Alderson-Rando Phantom, 60 thermoluminescent dosimeters [TLD]) was performed for dose measurements. Acquisition parameters were as follows: (i) pVFSE: $76.7 \mathrm{kV}, 57 \mathrm{~mA}, 0.9 \mathrm{Cu} \mathrm{mm}$, pulse rate/s 30; (ii) hrVFSE: $68.0 \mathrm{kV}, 362 \mathrm{~mA}, 0.2 \mathrm{Cu} \mathrm{mm}$, pictures $30 / \mathrm{s}$. The dose area product (DAP) indicated by the detector system and the radiation dose derived from the TLD measurements were analyzed. In a patient study, image quality was assessed qualitatively (5-point Likert scale, 5 = hrVFSE; two independent readers) and quantitatively (SNR) in 35 patients who subsequently underwent contrast-enhanced pVFSE and hrVFSE.

Results: Phantom measurements showed a dose reduction per picture of factor 25 for pVFSE versus hrVFSE images $(0.0025 \mathrm{mGy}$ versus $0.062 \mathrm{mGy})$. The DAP $\left(\mu \mathrm{Gym}^{2}\right)$ was 28.0 versus 810.5 (pVFSE versus hrVFSE) for an average examination time of $30 \mathrm{~s}$. Direct and scattered organ doses were significantly lower for pVFSE as compared to hrVFSE $(P<0.05)$. Image quality was rated $3.9 \pm 0.5$ for pVFSE versus the hrVFSE standard; depiction of the contrast agent $4.8 \pm 0.3$; noise $3.6 \pm 0.5(P<0.05)$; SNR calculations revealed a relative decreased of $43.9 \%$ for $p V F S E$ as compared to hrVFSE.
\end{abstract}

Conclusion: Pulsed VFSE is feasible, providing diagnostic image quality at a significant dose reduction as compared to hrVFSE.

\section{Keywords}

Head/neck, fluoroscopy, eating disorders, radiation effects, technology assessments

Date received: 28 September 2016; accepted: 10 November 2016

\section{Introduction}

Dysphagia is a widespread diagnosis with a great variety of underlying pathologies $(1,2)$, such as cerebrovascular events, neurodegenerative diseases, and cancer of the nasopharyngeal and upper gastrointestinal tract $(3,4)$. Video-fluoroscopic swallowing exams (VFSE) are recognized as the standard of reference for imaging disorders of the swallowing process $(5,6)$. In addition to the primary
'Department of Diagnostic and Interventional Radiology, Eberhard-Karls University, Tuebingen, Germany

${ }^{2}$ Department of Diagnostic and Interventional Radiology, Ludwig-Maximilians-University Munich, Munich, Germany

${ }^{3}$ Department of Radiooncology and Radiotherapy, Ludwig-MaximiliansUniversity Munich, Munich, Germany

\section{Corresponding author:}

Mike Notohamiprodjo, Department of Diagnostic and Interventional Radiology, University of Tuebingen, Hoppe-Seyler-Straße 3, 72076

Tuebingen, Germany.

Email: Mike.Notohamiprodjo@uni-tuebingen.de 
diagnosis, VFSE serve as a helpful diagnostic tool for therapy planning and follow-up evaluation $(7,8)$. For precise diagnosis, good contrast and high temporal resolution are required at a preferably low dose exposure (9).

Although frequently used in daily clinical routine, very few studies have been published on the radiation dose efficiency of these examinations $(10,11)$. In particular, little is known about the actual organ dose absorbed by radiosensitive organs $(12,13)$ given the fact that the applied dose during VFSE is usually indicated by the detector system as dose area product (DAP) or entrance skin dose (ESD) $(14,15)$. This dose value does not take into account that some tissues are more susceptible to radiation damage than others (13). However, this is important to know since the applied radiation dose can reach or exceed those of modern low-dose cervical CT protocols (16), especially when VFSE is repeatedly performed at a high temporal resolution to evaluate the swallowing capability of fluid and different food consistencies (e.g. mush, pudding, solid food). Therefore, phantom studies are necessary to provide reliable dose values in order to facilitate adequate risk stratification in favor of increased patient safety (17). Moreover, examination methods and technical equipment of fluoroscopic examinations were continuously developed during the last decades from analogue film-screen techniques to digital detector systems including last image hold capability, pulsed fluoroscopy, and improved beam filtration with no appropriate research evaluating the clinical value and feasibility of these latest hardware and software developments for VFSE $(9,18)$.

Therefore, the purpose of this study was to evaluate the effectiveness and feasibility of a modern flat-panel detector system with state-of-the-art low-dose digital pulsed protocols for reducing dose exposure of VFSE (pVFSE) as compared to high-resolution digital radiograph examinations (hrVFSE) serving as a standard of reference. Our hypothesis was that pVFSE allows for a significant dose reduction, especially in distant radiosensitive organs at a retained diagnostic image quality.

\section{Material and Methods}

This retrospective study received approval by the local ethics committee of the Ludwig-MaximiliansUniversity, Munich, Germany and written informed consent was waived.

\section{Detector system and protocols}

All examinations were performed with a multifunctional flat-panel detector system (Artis Zee MP, Siemens Healthcare, Erlangen, Germany) by the same radiologist (AP). A microprocessor-controlled high-frequency X-ray generator $(100 \mathrm{~kW}$ at $100 \mathrm{kV})$ with an automatic X-ray control system was used. Focal spot size was $0.3 / 0.6 / 1.0 \mathrm{~mm}$; nominal power $(=0 \mathrm{~W})$ of the $\mathrm{X}$-ray tube and power according to IEC $(=300 \mathrm{~W})$ was $18 / 52 / 100 \mathrm{~kW}$ respectively $15 / 40 /$ 80 ; maximal exposure voltage $125 \mathrm{kV}$.

Detector size was $30 \times 40 \mathrm{~cm}$ ( $48 \mathrm{~m}$ diagonal entrance plane; cesium iodide [CsI] scintillator, amorphous silicion [a-Si]) with a pixel size of $154 \times 154 \mu \mathrm{m}, 14$-bit digitization depth, and a spatial resolution of $3.25 \mathrm{LP} / \mathrm{mm}$. The resolution was $512 \times 512$ for $\mathrm{pVFSE}$ images and $1024 \times 1024$ for hrVFSE examinations. Preset algorithms were utilized to improve image quality in terms of noise, contrast, and brightness.

Standard protocol settings comprised copper filtering (pVFSE; $0.9 \mathrm{~mm}$; hrVFSE, $0.2 \mathrm{~mm}$ ) and a pulse/ frame rate of $30 \mathrm{images} / \mathrm{s}$. The pVFSE was operated with $76.7 \mathrm{kV}$ and $57 \mathrm{~mA}$ whereas $68.0 \mathrm{kV}$ and $362 \mathrm{~mA}$ were used for the hrVFSE examinations. To further reduce dose exposure, last image hold capability allowing for radiation-free collimator adjustment and object positioning was used when necessary to plan the further examination. The field of view (FOV) was chosen as small as possible.

\section{Phantom studies}

In our institution, hrVFSE was the clinical standard examination for patients with swallowing disorders. After the application of a new software package, pVFSE replaced hrVFSE as examination of choice due to its substantially reduced dose exposure. To systematically assess the differences in dose exposure between the two examination protocols, we used an anthropomorphic male Alderson-Rando Phantom (Humanoid Phantom, Type RT200, Humanoid Systems, Carson, CA, USA), which represents a standardized male patient $(175 \mathrm{~cm}$ height and $73.5 \mathrm{~kg}$ weight) and is considered equivalent to human body tissue in terms of X-ray absorption and scattering. Sixty thermoluminescent dosimeters (TLD, LiF (Lithiumfluorid), TLD Type-100, $1 \times 6 \mathrm{~mm}$, Harshaw, Chemical Company, Solon, OH, USA) were placed in relevant organs of interest comprising the salivary glands, the thyroid, the red bone marrow, and the esophagus in the direct radiation beam and the eyes, the thymus, the lungs, and the gonads in the scattered beam. A phantom scheme showing the TLD distribution is provided in Fig. 1.

For more reliable results, every organ was equipped with at least two TLD. In order to reduce measurement errors, images (pVFSE and hrVFSE) were acquired at a pulse rate of 30 images/s until a cumulative dose of $500 \mathrm{mGy}$ was indicated by the detector system. TLD were recalibrated before each measurement to ensure reliable dose values. Dose values for direct and 


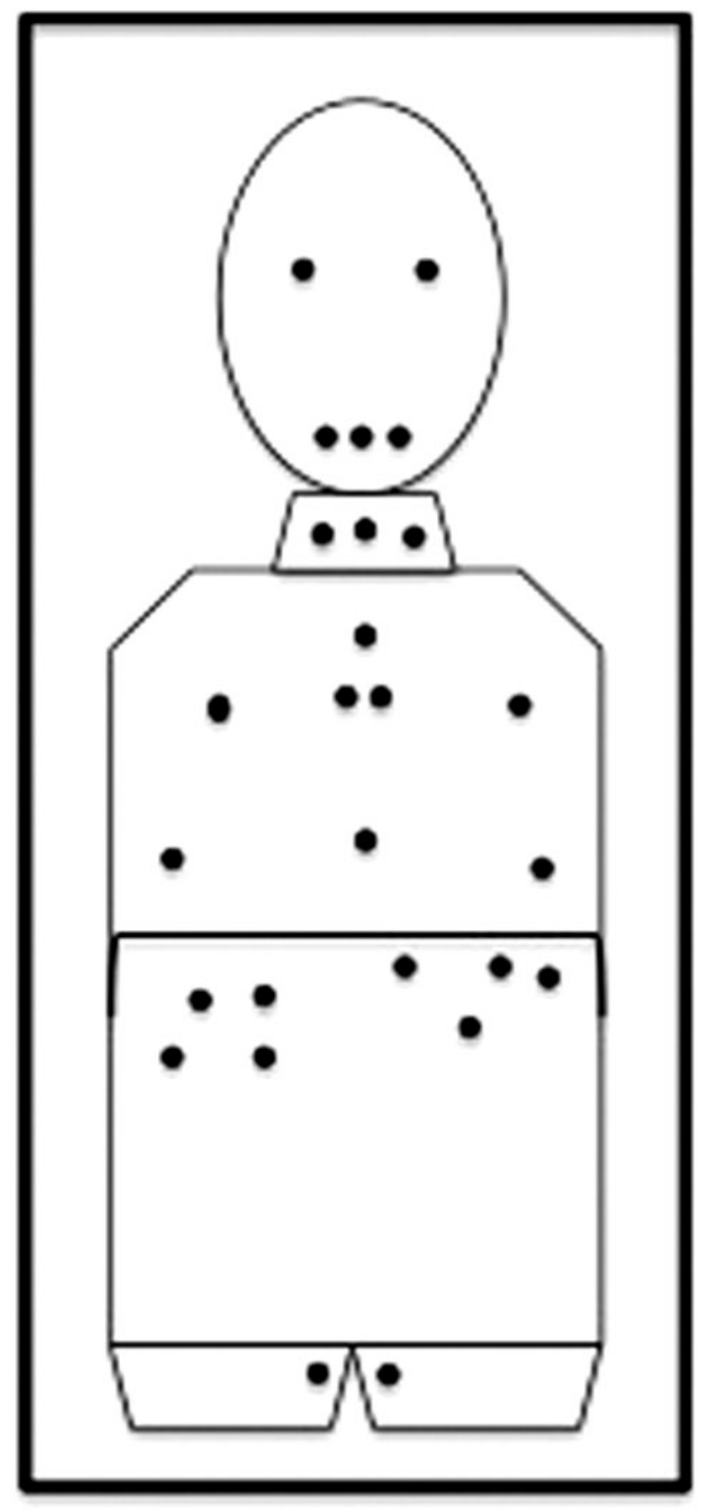

Fig. I. Thermoluminescent dosimeter distribution scheme of the Alderson Rando Phantom.

scattered organ dose (mGy) were calculated with the mean dose of all TLD placed in an individual organ $(\mathrm{n}=2-4)$. The presented values of the DAP $\left(\mu \mathrm{Gym}^{2}\right)$ and absorbed organ doses derived from the TLD measurements were calculated for the duration of an average examination observed in the patient study (see below). Exposure was performed with a tube distance of $35.5 \mathrm{~cm}$ to the phantom and a distance of $31.0 \mathrm{~cm}$ to the detector. The FOV involved the oral cavity and the oro-/hypopharynx (Fig. 2).

\section{Patient study}

In addition to the dose measurements in the phantom study, we performed a patient study to evaluate the image quality of the pVFSE protocol in clinical routine in comparison to the former hrVFSE standard protocol. For this reason, we retrospectively included 35 patients, who subsequently underwent contrastenhanced (Imeron 400, Bracco, Milan, Italy; Peritrast 400, Dr. Franz Köhler Chemie GmbH, Bensheim, Germany) hrVFSE before the software update and routine follow-up pVFSE after the software update. All examinations were clinically indicated for further evaluation of swallowing disorders and were performed by the same examiner. Exclusion criteria included incomplete examinations due to severe aspiration or poor physical condition of the patient and patients aged under 18 years.

Image quality analysis: All images were appraised on a dedicated workstation (syngoMMWP VE26A; Siemens Healthcare, Erlangen, Germany). Image quality was independently assessed by two radiologists (AP, MN) with 3 and 6 years of experience, respectively, in fluoroscopy. Images were evaluated in a random order and both readers were blinded to the clinical diagnosis and the type of examination protocol. All ratings used a five-point Likert scale comprising the following parameters: overall image quality; depiction of the contrast agent; subjective image noise. Per definition, qualitative image parameters were rated as 5 in hrVFSE examinations, indicating the most satisfying acquisition respectively the least artifacts, as this was the former routine clinical examination, thus serving as standard of reference for the pVFSE protocol.

Signal-to-noise ratio (SNR) and relative SNR differences $(\triangle \mathrm{SNR})$ were calculated to obtain objective image parameters by placing a region of interest (ROI; similar size (ca. $2 \mathrm{~cm}^{2}$ ) and position in pVFSE and hrVFSE) in the most homogeneous part of cervical soft tissue using the following equations:

$$
\begin{aligned}
& \mathrm{SNR}=\left(\text { mean signal }_{\mathrm{ROI}} / \text { mean } \mathrm{SD}_{\mathrm{ROI}}\right) \\
& \Delta \mathrm{SNR} \%=\left(1-\left[\mathrm{SNR}_{\mathrm{pVFSE}} / \mathrm{SNR}_{\mathrm{hrVFSE}}\right]\right) \times 100
\end{aligned}
$$

\section{Statistical analysis}

All statistical analyses were performed using SPSS (Version 22, IMB, Armonk, NY, USA). Qualitative reading scores were compared using the Wilcoxonsigned-rank test. For comparison of quantitative measurements paired t-test was conducted. $P$ values below 0.05 were assumed to indicate statistical significance. 


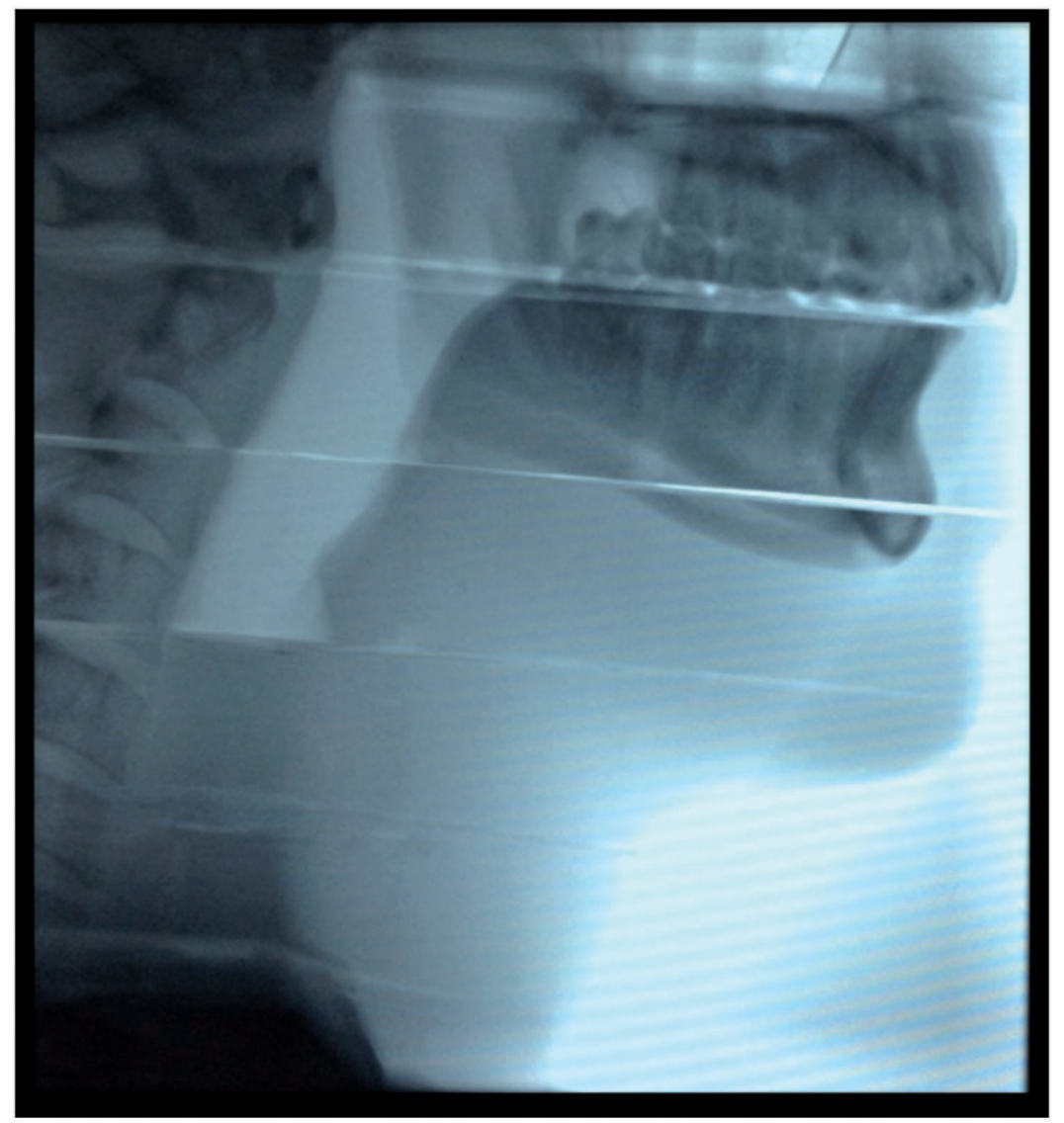

Fig. 2. FOV for image acquisition (pVFSE and hrVFSE) in the phantom study.

Inter-reader agreement was assessed by calculating Cohen's kappa, which was interpreted as follows: $<0.2$ poor correlation; $0.2-0.4$ fair correlation; $0.4-0.6$ moderate correlation; $0.6-0.8$ substantial correlation; and $>0.8$ excellent correlation.

\section{Results}

\section{Phantom study}

The cumulated phantom dose measurement showed a general dose reduction per picture of factor 25 for pVFSE versus hrVFSE images $(0.0025 \mathrm{mGy}$ versus $0.062 \mathrm{mGy})$.

To ease comparability and to provide reference values for clinical routine the presented results for both, DAP and direct as well as scattered radiation levels were calculated for an average acquisition time of $30 \mathrm{~s}$ due to the mean examination time of $34 \mathrm{~s}$ (range, 11-106s) in the patient study (see below). The resulting DAP $\left(\mu \mathrm{Gym}^{2}\right)$ indicated by the detector system was 28.0 for pVFSE and 810.5 for hrVFSE examinations (factor 29; thinsp; hrVFSE versus pVFSE).
Direct and scattered organ dose (mGy) was significantly lower $(P<0.05)$ for pVFSE versus hrVFSE images in all observed organs. As to be expected, highest dose exposure was measured in the direct beam (salivary glands, thyroid, esophagus, bone marrow). Scattered radiation dose decreased continuously with growing distance from the FOV, with highest radiation levels next to the FOV (eyes, thymus, lungs) and no measurable radiation dose in the gonads (male and female). Detailed results of the examined organs are provided in Table 1.

\section{Patient study}

VFSE with both examination protocols was successfully completed in all 35 patients (mean age, $66 \pm 16$ years; age range, 24-94 years; 19 men) and all patients were included in the final analysis. Unclear dysphagia was the most common diagnosis $(\mathrm{n}=12)$, followed by larynx carcinoma $(n=6)$ and oropharynx carcinoma $(n=6)$, Zenker's diverticulum $(n=4)$, esophagus carcinoma $(n=3)$, and others $(n=4)$. The average fluoroscopic acquisition time was $34 \mathrm{~s}$ (range, 11-106 s) 
depending on the physical condition and clinical diagnosis of the patient.

Image analysis: Overall image quality was rated $3.9 \pm 0.5$ for pVFSE as compared to the hrVFSE standard $(=5 ; P<0.05)$. The depiction of the contrast agent was graded with $4.8 \pm 0.3$ versus $5.0 \pm 0.0$; $P<0.05$ (pVFSE versus hrVFSE, respectively). Subjective image noise was markedly higher in pVFSE images in comparison to the hrVFSE standard $(3.6 \pm 0.5 ; \quad P<0.05)$ with substantial to excellent

Table I. Absorbed organ doses (mGy) for an average acquisition time of 30 seconds in direct and scattered radiation for hrVFSE and pVFSE.

\begin{tabular}{lll}
\hline & hrVFSE & PVFSE \\
\hline Scattered radiation (mGy) & & \\
$\quad$ Eyes & 2.43 & 0.11 \\
Thymus & 2.01 & 0.07 \\
Lungs & 1.38 & 0.05 \\
Gonads male & 0 & 0 \\
Gonads female & 0 & 0 \\
Direct radiation (mGy) & & \\
Salivary glands & 39.00 & 1.68 \\
$\quad$ Thyroid & 33.03 & 1.29 \\
Esophagus & 23.91 & 1.02 \\
Bone marrow & 3.03 & 0.17 \\
\hline
\end{tabular}

inter-reader agreement for all evaluated parameters (0.68-0.91).

SNR calculations revealed significantly lower values for the pVFSE $(48.0 \pm 22)$ as compared to the hrVFSE images $(88.5 \pm 36.6 ; P<0.001)$ resulting in a $\triangle \mathrm{SNR}$ of $43.9 \pm 19.1 \%$.

Exemplary images of the most common diagnoses are provided in Figs. 3-5.

\section{Discussion}

In this study, we evaluated the clinical value and feasibility of the latest technical advances for videofluoroscopic swallowing exams. We could demonstrate that low-dose digital pulsed protocols acquired with a modern flat-panel detector system allow for a significant dose reduction while retaining diagnostic image quality.

These are important results considering the frequent use in daily clinical routine $(19,20)$. In particular, patients with an above-average examination time due to passage disorders or patients repeatedly examined in order to assess postoperative outcome can cumulate a considerably high radiation dose with conventional hrVFSE protocols, particularly when different food consistencies are evaluated with a consequently prolonged examination time. In this context, our results indicate that digital pulsed VFSE using state-of-theart low-dose protocols is an effective approach to reduce dose exposure and seems a valuable

(a)

(b)

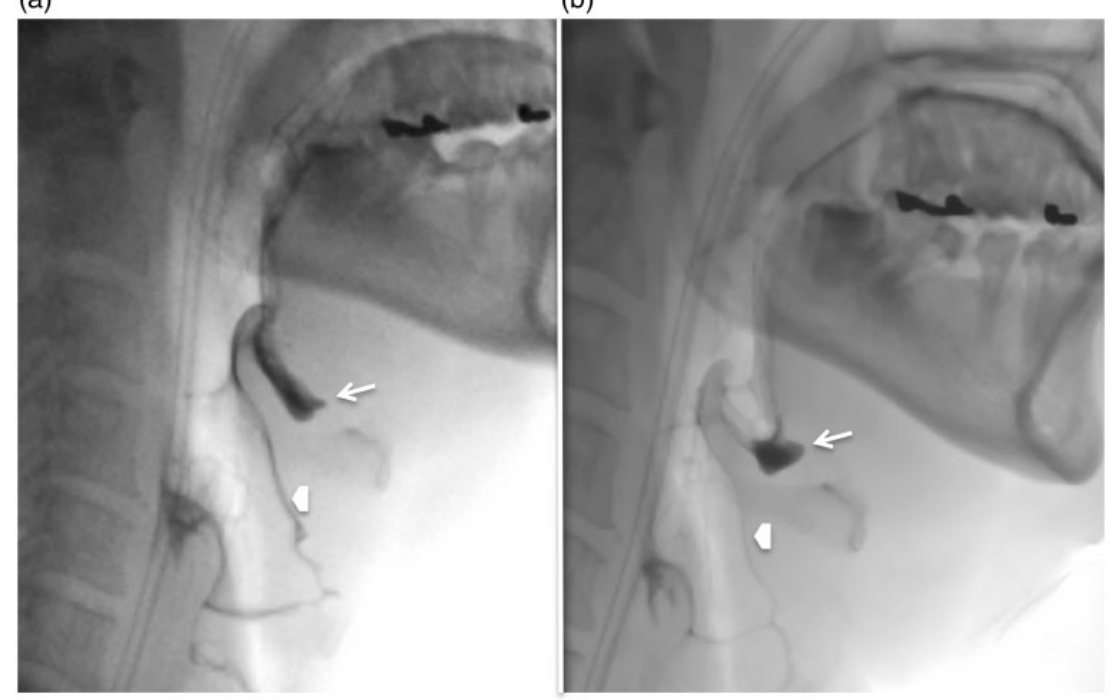

Fig. 3. PVFSE (a) and hrVFSE (b) images of a 52-year-old woman with dysphagia and recurrent aspirations post stroke. Images after oral contrast agent administration show contrast agent pooling superior to the epiglottis (arrow) and moderat intratracheal aspiration (arrow head) similarly detecable in PVFSE and hrVFSE. 
(a)

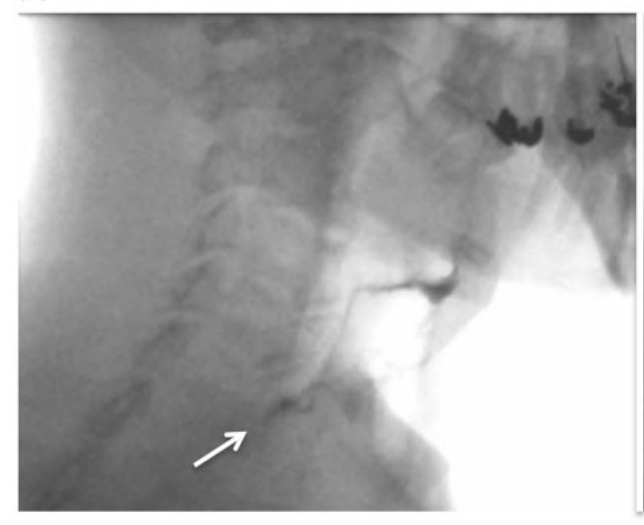

(b)

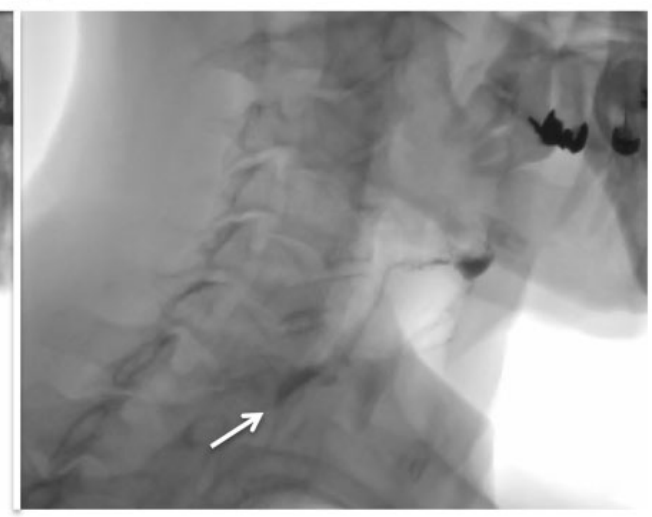

Fig. 4. pVFSE (a) and hrVFSE (b) images of a 69-year-old male patient with a small diverticulum in the proximal esophagus (arrow) similarly detectable in pVFSE and hrVFSE images.

alternative to replace standard of reference high-resolution VFSE examinations.

As expected and shown in our phantom study, organs in the direct beam showed the highest radiation levels whereas scattered dose exposure in distant organs is negligible low. In the gonads, for instance, no dose exposure could be measured at all. Thus, there appears no significant risk of gonadal radiation damage during pVFSE, even if frequent examinations should be necessary. Our results are consistent with Iwai et al., who also evaluated organ doses of swallowing examinations in a phantom study (12). Due to their different detector system and examination protocols the results cannot be compared absolutely; however, the distribution pattern of the radiation dose was similar as in our study. Others, like Zammit-Maempel (5) or Kim et al. (10), only estimated the total effective dose of VFSE using standardized Monte-Carlo derived conversion factors, however, at the cost of a remaining uncertainty regarding the actual dose absorbed. Nevertheless, this is a commonly used approach given that up till now the DAP is the standard of reference to quantify the dose exposure of fluoroscopic examinations. However, this dose value does not consider tissue specific radiation susceptibility $(13,21)$ and the DLP-derived estimation of the effective dose lacks the information of individual organ doses, although they are considered to provide the most detailed insight in the radiation burden of the patient (22). Therefore, the results of our phantom measurements presented in this study may serve as an orientation of the average organ dose exposure during a standard VFSE.

The results of our patient study revealed that lowdose pVFSE provide a sufficient image quality for a reliable diagnosis of all evaluated indications as compared to the hrVFSE reference standard although the relative SNR of pVFSE decreased by approximately $45 \%$. However, this had no relevant effect on diagnostic performance in clinical routine, since all pVFSE were rated as diagnostic and no additional hrVFSE or follow-up examination due to insufficient image quality was necessary to allow for an unequivocal diagnosis. Therefore, we consider low-dose pVFSE as a promising approach to increase patient safety by substantially reducing dose exposure without sacrificing a satisfying image quality. As mentioned above, this is of great importance, since frequent examinations can result in a high cumulative radiation dose with the risk of long-term radiation effects (23). Supported by these results, we have replaced the former hrVFSE reference standard by the pVFSE protocol as routine clinical examination of choice in patients with swallowing disorders.

As mentioned above, technical equipment has been continuously developed in the last decades with no appropriate research evaluating quality standards of both examination methods and hardware devices for VFSE. Relevant advances allowing for reduced dose exposure are, among others, pulsed image acquisition, last image-hold capability, automatic dose control, and beam filtering $(24,25)$, which were also applied in this study but have yet only been evaluated in the setting of heart catheter examinations and in pediatric radiology $(24,26)$. With this study we provide an update and overview of the effectiveness and clinical feasibility of the latest hardware and software equipment for standard VFSE examinations in clinical routine.

Some limitations of this study need to be addressed. First, it is well-known that fluoroscopic examinations are highly dependent to individual operator practice regarding duration, accurate superimposition, and 


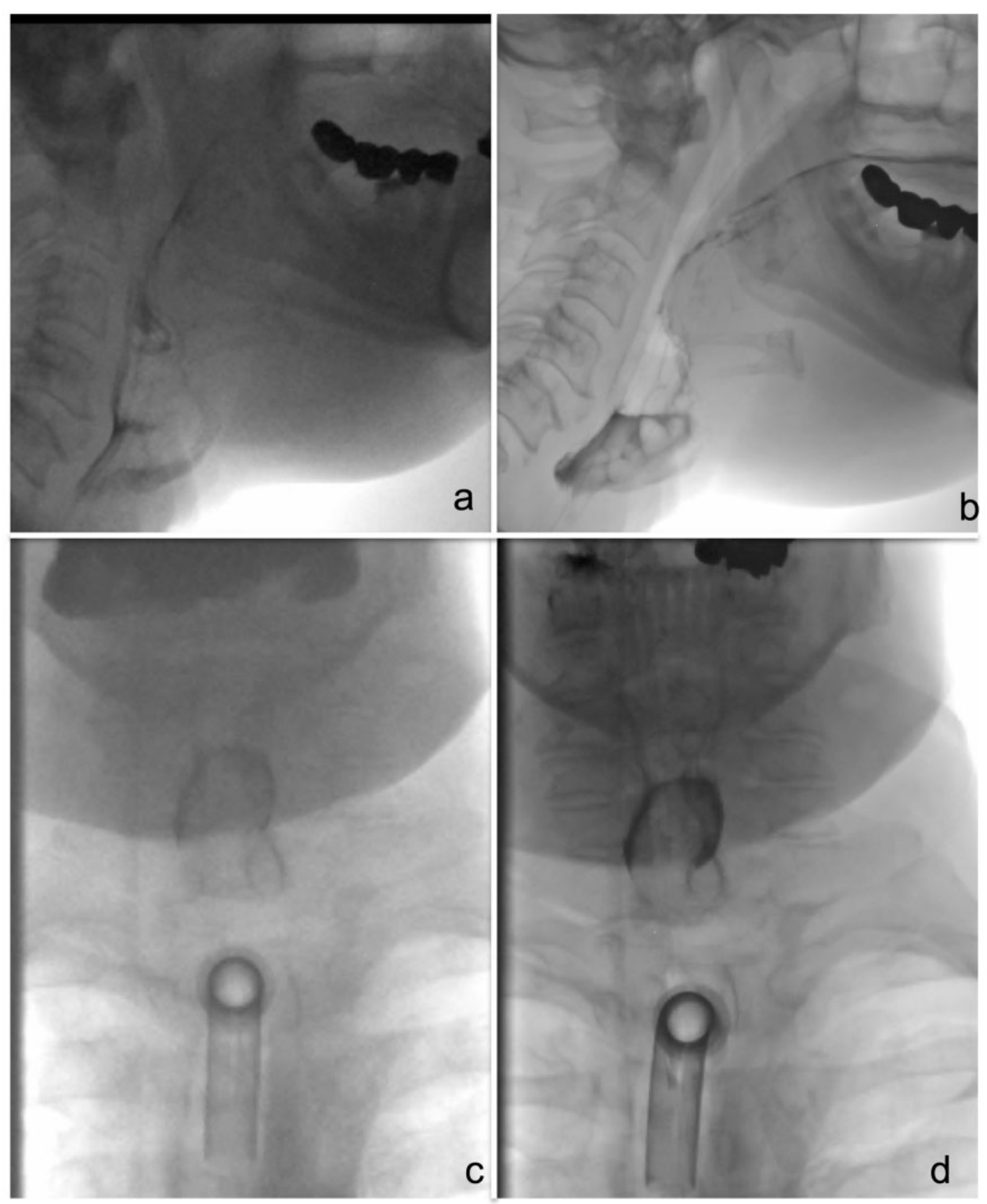

Fig. 5. pVFSE (a, c) and hrVFSE (b, d) images of a 73-year-old male patient post larnygectomie due to larynx carcinoma. Depiction of contrast agent was rated equivalent in PVFSE and hrVFSE images whereas image nosie was rated higher in pVFSE (a, c) images.

correct patient distance to the tube with direct effects on the resulting dose exposure (13). To rule out these inter-operator effects on the tested examination protocols, we only included examinations obtained by a single examiner and normalized the dose results for an average examination time of $30 \mathrm{~s}$. However, these results may only serve as a general orientation, because no information about inter-observer performance was taken into account. Furthermore, it is not possible to simulate the administration of contrast agent in the Alderson-Rando Phantom to evaluate its effect on the radiation dose, whereas dose measurements in the patient study were performed after contrast agent administration. Additionally, the phantom dose measurements were only performed once and therefore no information about re-test repeatability is available and it is not possible to differentiate the individual contribution of the different dose-saving methods and software algorithms to the overall dose reduction achieved by the pVFSE protocol.

In conclusion, pulsed VFSE with a modern flatpanel detector system provides diagnostic image quality at a significant dose reduction as compared to standard of reference hrVFSE. Scattered radiation levels in distant radiosensitive organs are negligible low with no severe risk of radiation damage. 


\section{Declaration of Conflicting Interests}

The author(s) declared no potential conflicts of interest with respect to the research, authorship, and/or publication of this article.

\section{Funding}

The author(s) received no financial support for the research, authorship, and/or publication of this article.

\section{References}

1. Jaffer NM, Ng E, Au FW, et al. Fluoroscopic evaluation of oropharyngeal dysphagia: anatomic, technical, and common etiologic factors. Am J Roentgenol 2015;204: 49-58.

2. Rofes L, Arreola V, Almirall J, et al. Diagnosis and management of oropharyngeal Dysphagia and its nutritional and respiratory complications in the elderly. Gastroenterol Res Pract 2011;2011:818979.

3. Palmer JB, Drennan JC, Baba M. Evaluation and treatment of swallowing impairments. Am Fam Physician 2000;61:2453-2462.

4. Aslam M, Vaezi MF. Dysphagia in the elderly. Gastroenterol Hepatol (NY) 2013;9:784-795.

5. Zammit-Maempel I, Chapple CL, Leslie P. Radiation dose in videofluoroscopic swallow studies. Dysphagia 2007;22:13-15.

6. Crawley MT, Savage P, Oakley F. Patient and operator dose during fluoroscopic examination of swallow mechanism. Br J Radiol 2004;77:654-656.

7. Moro L, Cazzani C. Dynamic swallowing study and radiation dose to patients. Radiol Med 2006;111:123-129.

8. Yang CJ, Roh JL, Choi KH, et al. Pretreatment dysphagia inventory and videofluorographic swallowing study as prognostic indicators of early survival outcomes in head and neck cancer. Cancer 2015;121:1588-1598.

9. Barkhof F, David E, de Geest F. Comparison of filmscreen combination and digital fluorography in gastrointestinal barium examinations in a clinical setting. Eur J Radiol 1996;22:232-235.

10. Kim HM, Choi KH, Kim TW. Patients' radiation dose during videofluoroscopic swallowing studies according to underlying characteristics. Dysphagia 2013;28:153-158.

11. Martin-Harris B, Logemann JA, McMahon S, et al. Clinical utility of the modified barium swallow. Dysphagia 2000;15:136-141.

12. Iwai K, Hashimoto K, Nishizawa K, et al. Evaluation of effective dose from a RANDO phantom in videofluorography diagnostic procedures for diagnosing dysphagia. Dentomaxillofac Radiol 2011;40:96-101.

13. Wright RE, Boyd CS, Workman A. Radiation doses to patients during pharyngeal videofluoroscopy. Dysphagia 1998;13:113-115.

14. Chau KH, Kung CM. Patient dose during videofluoroscopy swallowing studies in a Hong Kong public hospital. Dysphagia 2009;24:387-390.

15. Schubert R. What is dose area product? Radiol Technol 1995;66:329-330.

16. Campbell MJ, Sicuro P, Alseidi A, et al. Two-phase (lowdose) computed tomography is as effective as 4D-CT for identifying enlarged parathyroid glands. Int J Surg 2015; 14:80-84.

17. Miksys N, Gordon CL, Thomas K, et al. Estimating effective dose to pediatric patients undergoing interventional radiology procedures using anthropomorphic phantoms and MOSFET dosimeters. Am J Roentgenol 2010;194:1315-1322.

18. Lin PJ. Technical advances of interventional fluoroscopy and flat panel image receptor. Health Phys 2008;95: 650-657.

19. Ramsey DJ, Smithard DG, Kalra L. Early assessments of dysphagia and aspiration risk in acute stroke patients. Stroke 2003;34:1252-1257.

20. Shem KL, Castillo K, Wong SL, et al. Diagnostic accuracy of bedside swallow evaluation versus videofluoroscopy to assess dysphagia in individuals with tetraplegia. PM R 2012;4:283-289.

21. Kamiya K, Ozasa K, Akiba S, et al. Long-term effects of radiation exposure on health. Lancet 2015;386:469-478.

22. Samei E, Tian X, Segars WP. Determining organ dose: the holy grail. Pediatr Radiol 2014;44(Suppl. 3): 460-467.

23. Brenner DJ, Doll R, Goodhead DT, et al. Cancer risks attributable to low doses of ionizing radiation: assessing what we really know. Proc Natl Acad Sci U S A 2003;100: 13761-13766.

24. Nickoloff EL, Lu ZF, Dutta A, et al. Influence of flatpanel fluoroscopic equipment variables on cardiac radiation doses. Cardiovasc Intervent Radiol 2007;30: 169-176.

25. Heidbuchel H, Wittkampf FH, Vano E, et al. Practical ways to reduce radiation dose for patients and staff during device implantations and electrophysiological procedures. Europace 2014;16:946-964.

26. Kuon E, Weitmann K, Hummel A, et al. Latest-generation catheterization systems enable invasive submillisievert coronary angiography. Herz 2015;40(Suppl. 3): 233-239. 